\title{
"Kimura-first" strategy for robotic spleen-preserving distal pancreatectomy: experiences from 61 consecutive cases in a single institution
}

\author{
Xianchao Lin", Ronggui Lin", Fengchun Lu, Yuanyuan Yang, Congfei Wang, Haizong Fang, Heguang Huang \\ Department of General Surgery, Fujian Medical University Union Hospital, Fuzhou, Fujian, China \\ Contributions: (I) Conception and design: X Lin, H Huang; (II) Administrative support: F Lu; (III) Provision of study materials or patients: All \\ authors; (IV) Collection and assembly of data: Y Yang, C Wang, H Fang; (V) Data analysis and interpretation: R Lin, H Fang; (VI) Manuscript \\ writing: All authors; (VII) Final approval of manuscript: All authors. \\ "These authors contributed equally to this work. \\ Correspondence to: Heguang Huang. Department of General Surgery, Fujian Medical University Union Hospital, 29 Xinquan Road, Fuzhou 350001, \\ China. Email: Heguanghuang2@163.com.
}

Background Robotic spleen-preserving distal pancreatectomy (RSPDP) is an ideal procedure for benign
and low-grade malignant tumors in the distal pancreas, and two splenic preservation techniques (the Kimura
and Warshaw techniques) can be used for RSPDP. This study aimed to evaluate the feasibility and safety of
the "Kimura-first" strategy for RSPDP and to investigate the risk factors affecting the preservation of the
spleen and splenic vessels.

Methods: The electronic medical records of patients who underwent robotic distal pancreatectomy (RDP) between October 2016 and December 2019 at our institution were retrospectively reviewed. Univariate and multivariate analyses were conducted to identify the risk factors influencing preservation of the spleen and splenic vessels during RDP.

Results: Sixty-one patients scheduled for RSPDP who received RDP were included in this study [Kimura technique, 41 patients; Warshaw technique, 11 patients; and robotic distal pancreatectomy with splenectomy (RDPS), 9 patients]. The overall splenic preservation rate with RDP was 85.2\% (52/61). The preservation rate of splenic vessels with the Kimura technique with RSPDP was 78.8\% (41/52). The RSPDP group had remarkably less estimated blood loss (EBL; median 50 vs. $300 \mathrm{~mL}, \mathrm{P}=0.000)$ and a lower morbidity rate $(13.5 \%$ vs. $44.4 \%, \mathrm{P}=0.047$ ) than the RDPS group. The logistic regression models showed that obvious splenic vessel compression by the tumor was an independent risk factor for splenic vessel preservation with RSPDP (OR 0.021, 95\% CI: 0.002-0.271, P=0.003) and RDP (OR 0.019, 95\% CI: 0.002-0.176, P=0.000).

Conclusions: The "Kimura-first" strategy is feasible and safe for RSPDP, with high rates of splenic and splenic vessel preservation. Obvious splenic vessel compression by the tumor can be used as a predictor of splenic vessel preservation with planned RDP.

Keywords: Robotic; distal pancreatectomy; splenic preservation; splenectomy

Submitted Jul 22, 2020. Accepted for publication Sep 30, 2020.

doi: $10.21037 /$ gs-20-576

View this article at: http://dx.doi.org/10.21037/gs-20-576

\section{Introduction}

Minimally invasive distal pancreatectomy (MIDP) has been widely accepted as a routine procedure for benign and malignant tumors of the pancreatic body and tail (1,2). MIDP mainly includes laparoscopic distal pancreatectomy (LDP) and robotic distal pancreatectomy (RDP) (3). A recent multicenter randomized controlled trial (RCT) by de Rooij et al. showed that MIDP had 
less blood loss than open distal pancreatectomy (ODP) and comparable rates of postoperative complications and costs (4). In addition, compared with ODP, MIDP shortened the time to functional recovery, decreased the incidence of delayed gastric emptying (DGE) and increased quality of life.

The spleen is often resected in distal pancreatectomy (DP) due to severe adhesion between the splenic vessels and the pancreas or tumor, as well as for the radical resection requirement of malignant pancreatic neoplasms. Currently, spleen-preserving procedures are advocated for benign and low-grade malignant pancreatic tumors to avoid sacrificing the spleen in DP (5-7). The techniques of spleen-preserving distal pancreatectomy (SPDP) mainly include the Kimura technique (8), with complete preservation of the splenic artery and vein, and the Warshaw technique (9), with segmental resection of the splenic vessels and preservation of the left gastroepiploic vessels and the short gastric vessels for the spleen supply.

Spleen-preserving MIDP has been increasingly performed worldwide in recent decades (10). Several studies have reported the superiority of the robotic system compared to laparoscopic procedures for splenic preservation in DP (11-13). In contrast to laparoscopic procedures, the robotic system provides a magnified three-dimensional operative view, as well as more stable, flexible, and precise manipulation. The advantages of the robotic system make it safe and feasible when separating splenic vessels from the distal pancreas or tumor, dividing the branches of the splenic vessels as they course into the pancreas, and controlling unexpected bleeding of the splenic vessels. Accordingly, RDP has a relatively higher rate of splenic preservation than LDP (14). Liu et al. reported that RDP had a lower rate of conversion, a shorter postoperative stay, and higher rates of splenic and splenic vessel preservation than LDP (15).

In the present study, we conducted a retrospective analysis of patients with benign and low-grade malignant pancreatic tumors who underwent RDP with the "Kimurafirst" three-step strategy for splenic preservation. This study aimed to evaluate the feasibility and safety of the "Kimura-first" strategy for robotic spleen-preserving distal pancreatectomy (RSPDP) and to investigate the risk factors affecting the preservation of the spleen and splenic vessels. We present the following article in accordance with the STROBE reporting checklist (available at http://dx.doi. org/10.21037/gs-20-576).

\section{Methods}

Patients who underwent RDP at our institution between October 2016 and December 2019 were enrolled in this study. Patients planned for RSPDP with diagnoses of benign and low-grade malignant pancreatic tumors were included. Patients with presumed pancreatic cancer were excluded because they required procedures combined with radical splenectomy. The study was conducted in accordance with the Declaration of Helsinki (as revised in 2013). The study protocol was approved by the ethics committee of Fujian Medical University Union Hospital (2020KY0107). Written informed consent was obtained from the patients for publication of this manuscript and any accompanying images.

The clinicopathological characteristics and perioperative and postoperative outcomes of the patients were retrospectively collected from electronic medical records. The clinicopathologic characteristics included age, sex, body mass index (BMI), tumor size, tumor location, radiographic appearance, splenic vessel compression by the tumor (Figure 1) and pathological diagnosis. The perioperative and postoperative outcomes included the operative time, estimated blood loss (EBL), conversion, postoperative length of stay (LOS), morbidity, clinically relevant postoperative pancreatic fistula (CR-POPF) and mortality.

\section{Definitions}

CR-POPF is defined by the International Study Group of Pancreatic Surgery (ISGPS) as an amylase level in the drainage fluid $>3$ times the upper limit of normal serum amylase, with a POPF-associated clinically relevant condition (16). CR-POPF is classified as grade B or grade $\mathrm{C}$, and the latter grade often results in life-threatening complications.

\section{Operative technique}

All surgeries were completed by an experienced surgeon with the da Vinci Si HD Surgical System (Intuitive Surgical Inc., USA). The patient was placed in a supine position with slight right tilt after general anesthesia. A five-trocar technique was adopted for robotic manipulation (Figure 2).

Splenic preservation was attempted with the "Kimurafirst" three-step strategy, and the Kimura technique 

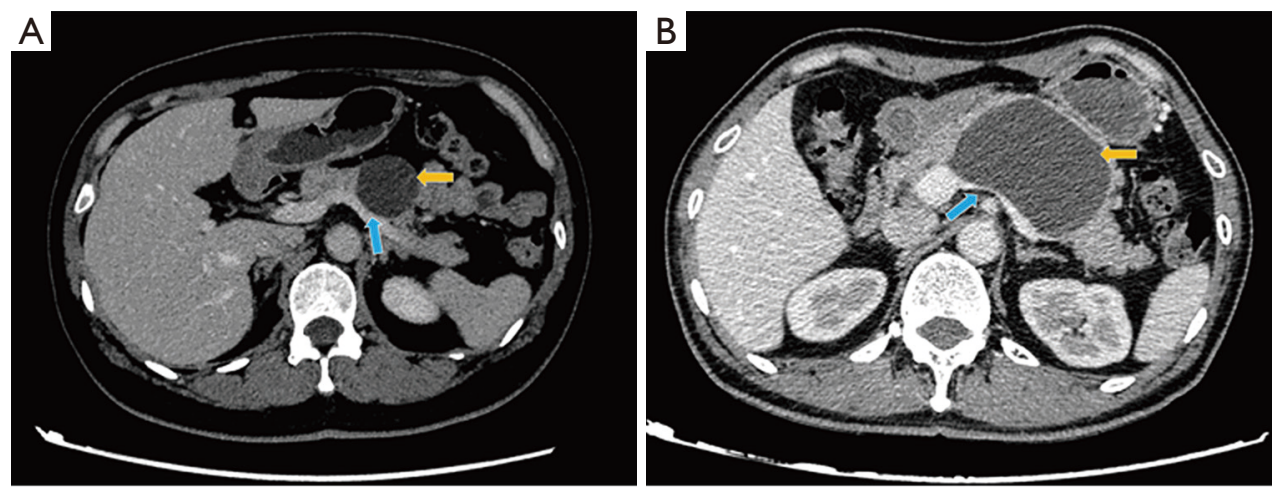

Figure 1 Representative computed tomography images of the relationship between pancreatic tumors and splenic vessels. (A) A cystic tumor in the pancreatic body (orange arrow) with no compression of the splenic vessels (blue arrow). (B) A cystic tumor in the pancreatic body and tail (orange arrow) with obvious compression of the splenic vessels (blue arrow).

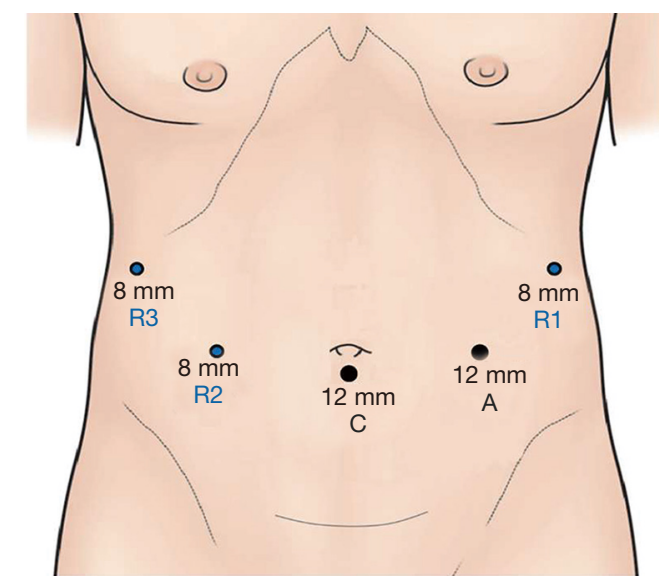

Figure 2 Trocar placements in RDP.

was considered the first choice. For the Kimura procedure (8) (Figure 3), the left gastroepiploic vessels and the short gastric vessels were initially preserved once the procedure was converted to the Warshaw procedure. The common hepatic artery (CHA) and the left gastric artery (LGA) were dissected in patients in whom the tumors were located close to the superior mesenteric vein (SMV). The distal pancreas was sufficiently dissected, and the splenic artery (SA) and splenic vein (SV) were identified and well protected. After sufficient dissection between the splenic vessels and pancreas was achieved, a $60-\mathrm{mm}$ linear cutter stapler (Echelon, USA) was used to transect the pancreas 2 $\mathrm{cm}$ to the right of the pancreatic tumor. The distal pancreas was separated from the splenic vessels in a medial-to-lateral direction. The branches of the splenic vessels coursing into the pancreas were divided with vascular clips or 5-0 Prolene sutures. After resection of the distal pancreas, the splenic vessels were carefully examined, and potential bleeding was controlled with 5-0 Prolene sutures. A drainage tube was routinely placed beside the stump of the pancreas. Frozen sectioning of the pancreatic tumor was routinely performed, and the spleen was resected in patients with a malignant tumor.

The Warshaw procedure (9) (Figure 4) was performed if the splenic vessels could not be safely preserved. After sufficient dissection of the splenic vessels and the distal pancreas, the splenic vessels and the distal pancreas were then divided. The splenic vessels and the distal pancreas were gradually dissected toward the splenic hilum. The branches of the vessels in the splenic hilum, the left gastroepiploic vessels and the short gastric vessels were well protected. The splenic vessels near the splenic hilum were then divided with an Ethilon stapler or vascular clips. After resection, the spleen was checked and resected in a patient with a greatly extending infarction. A drainage tube was routinely placed beside the stump of the pancreas.

If the Kimura and Warshaw procedures were not applicable, if there was insufficient blood supply to the spleen after the Warshaw procedure, or if the frozen sections were positive for malignancy, robotic distal pancreatectomy with splenectomy (RDPS) was performed. The short gastric vessels were divided, followed by sufficient dissection of the spleen. The splenic vessels were divided, and the pancreas was subsequently transected. With a routine drainage tube beside the stump of the pancreas, another drainage tube was placed beneath the diaphragm. 

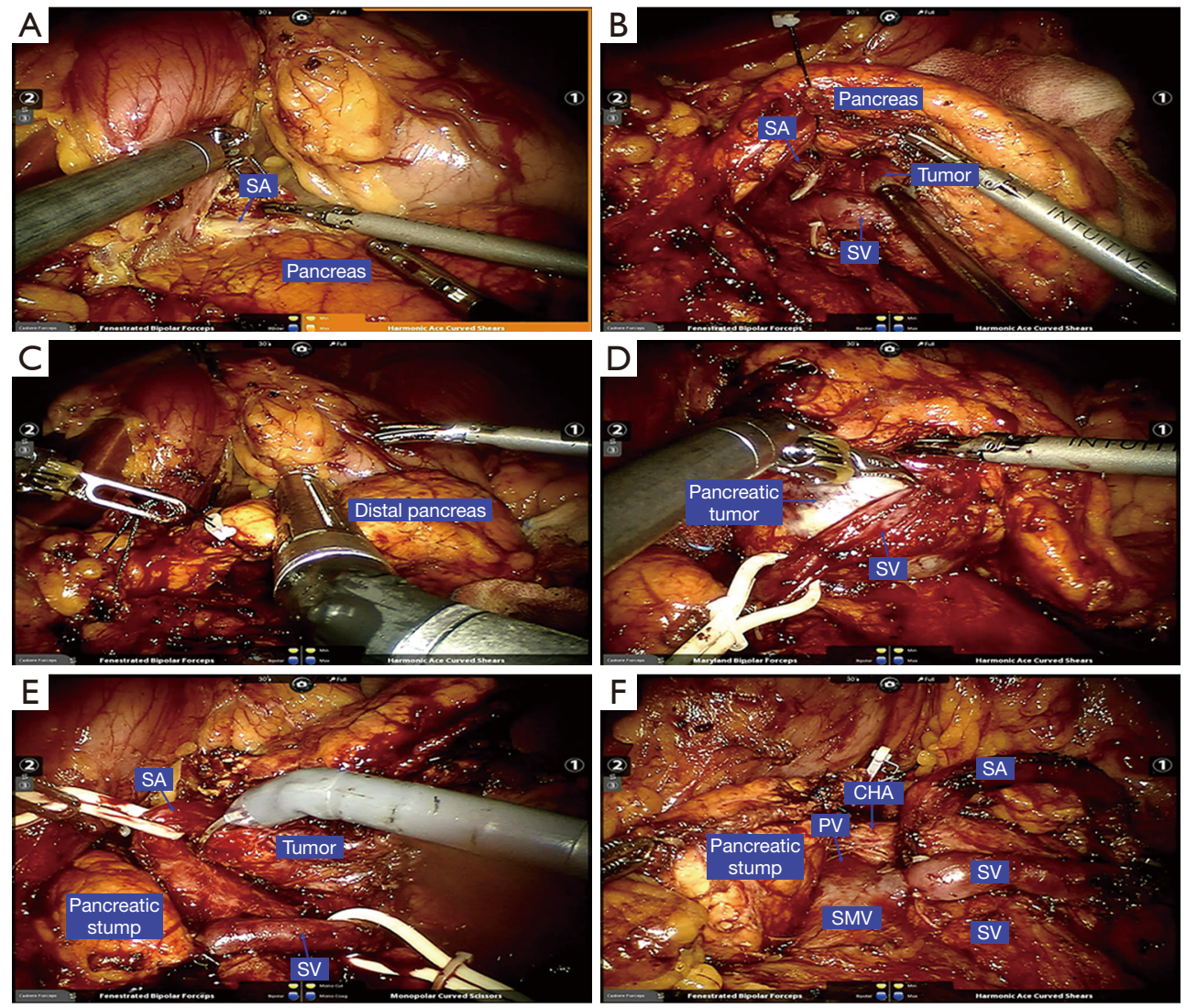

Figure 3 Intraoperative images of RSPDP with the Kimura technique. (A) Exposure of the SA at the superior border of the pancreatic neck. (B) Suspension of the pancreas after sufficient dissection of the postpancreatic space in front of the SV. (C) Transection of the pancreas $2 \mathrm{~cm}$ to the right of the pancreatic tumor with a $60-\mathrm{mm}$ linear cutter stapler. (D) Careful separation of the adhesions between the pancreatic tumor and the SV. (E) Separation of the adhesions between the pancreatic tumor and the SA. (F) Anatomic structures after resection of the distal pancreas with preservation of the splenic vessels. SA, splenic artery; SV, splenic vein; SMV, superior mesenteric vein; PV, portal vein; CHA, common hepatic artery.

\section{Statistical analysis}

Continuous variables are expressed as the mean and standard deviation or median and interquartile range (IQR), and categorical variables are reported as percentages and frequencies. Continuous variables were compared with independent-samples $t$-tests or nonparametric Mann-Whitney $U$ tests. Categorical variables were compared with the chi-square test or Fisher's exact test. Logistic regression was used to conduct univariate and multivariate analyses to identify risk factors for RDP with splenic or splenic vessel preservation. All variables with $\mathrm{P}<0.250$ in the univariate analysis were included in the logistic regression model. A $\mathrm{P}$ value $<0.05$ was considered statistically significant.
Statistical analysis was conducted with SPSS software (version 21.0, IBM).

\section{Results}

A total of 71 patients with planned RDP were enrolled in this study between October 2016 and December 2019. Ten patients were excluded because of scheduled RDPS for presumed pancreatic cancer. Of the remaining 61 patients, splenic preservation was achieved in 52 patients, and splenectomy was performed in 9 patients (as shown in Figure 5). Of the 52 patients, the Kimura procedure was applied in 41 patients, and the Warshaw procedure was applied in 11 patients. Of the 9 patients who received RDPS, one who underwent resection with the Warshaw technique 

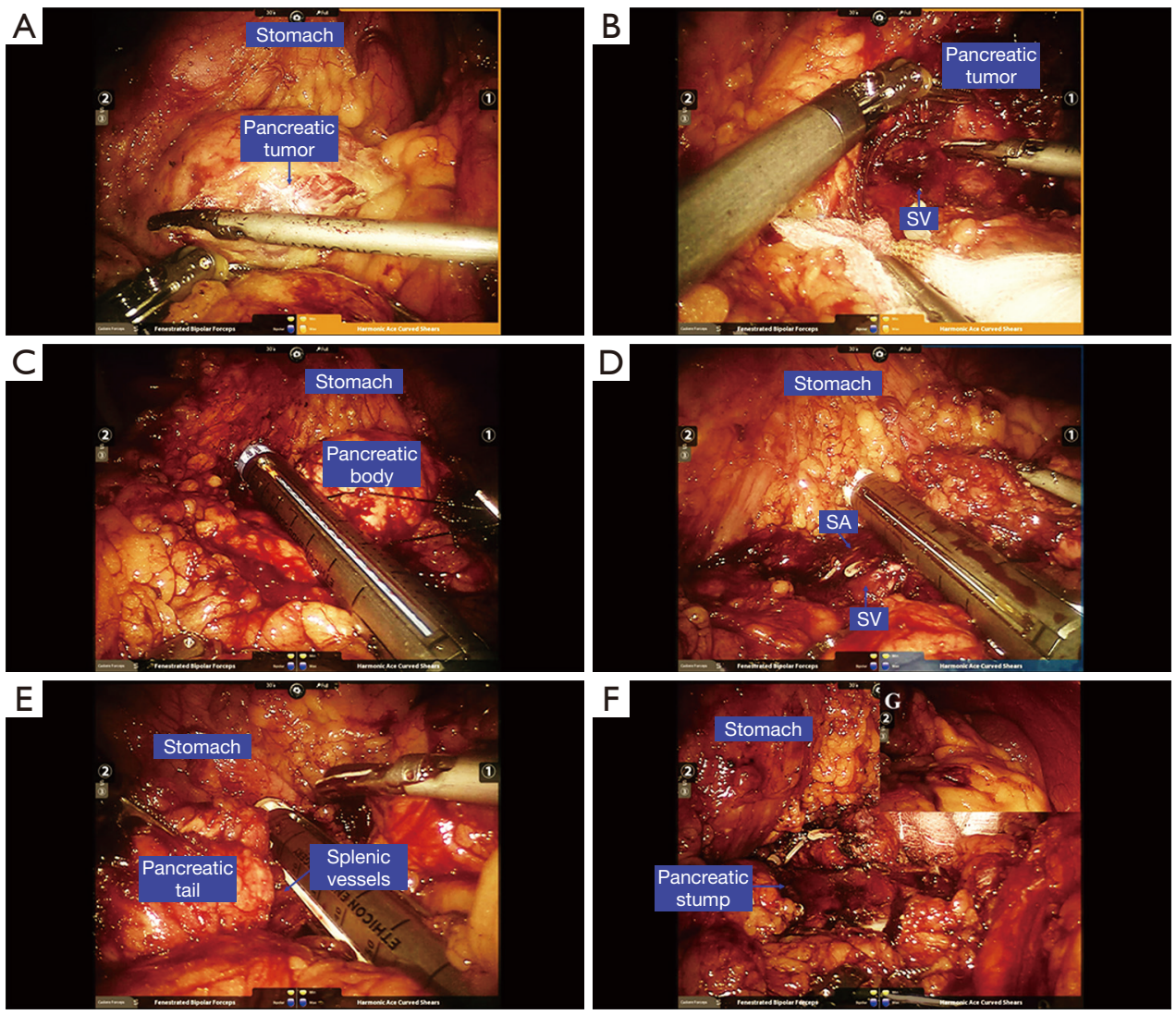

Figure 4 Intraoperative images of RSPDP with the Warshaw technique. (A) Dissection at the inferior border of the pancreas. (B) Separation of the postpancreatic space in front of the SV. (C) Transection of the pancreas $2 \mathrm{~cm}$ to the right of the pancreatic tumor with a 60 -mm linear cutter stapler. (D) Division of the splenic vessels near the root with a 60 -mm linear cutter stapler. (E) Division of the splenic vessels near the splenic hilum with a $60-\mathrm{mm}$ linear cutter stapler. (F) Corresponding structures after resection of the distal pancreas with sufficient blood supply to the spleen (G). SV, splenic vein; SA, splenic artery.

ultimately received splenectomy because of a greatly extending splenic infarction.

\section{Comparison between RSPDP and RDPS}

The clinicopathologic characteristics and perioperative and postoperative factors of the 61 patients who underwent RDP with or without spleen preservation are indicated in Table 1 (RSPDP vs. RDPS). Age, sex, BMI, tumor location, radiographic appearance and pathological diagnosis were comparable between the RSPDP and RDPS groups. The RDPS group had significantly higher rates of tumors $\geq 6$ $\mathrm{cm}(55.6 \%$ vs. $21.1 \%, \mathrm{P}=0.045)$ and obvious splenic vessel compression by the tumor $(66.7 \%$ vs. $23.1 \%, \mathrm{P}=0.015)$ than the RSPDP group. The RSPDP group had remarkably less $\mathrm{EBL}$ (median 50 vs. $300 \mathrm{~mL}, \mathrm{P}=0.000$ ) and a lower morbidity rate $(13.5 \%$ vs. $44.4 \%, \mathrm{P}=0.047)$ than the RDPS group. The operative time, conversion rate, LOS, and POPF rate between the two groups were not significantly different $(\mathrm{P}>0.05)$. There were no cases of postoperative intra-abdominal hemorrhage, reoperation, or mortality in the two groups.

\section{Comparison between the Kimura and Warshaw technique}

Table 2 describes the clinicopathologic characteristics and perioperative and postoperative factors of the 52 patients who underwent RSPDP (the Kimura technique $v$ s. the Warshaw technique). The radiographic appearance of the two groups was significantly different $(\mathrm{P}=0.000)$, and there was a larger proportion of cystic tumors in the Kimura group than in the Warshaw group. The Warshaw group had a notably higher rate of obvious splenic vessel compression by the tumor than the Kimura group (63.6\% 


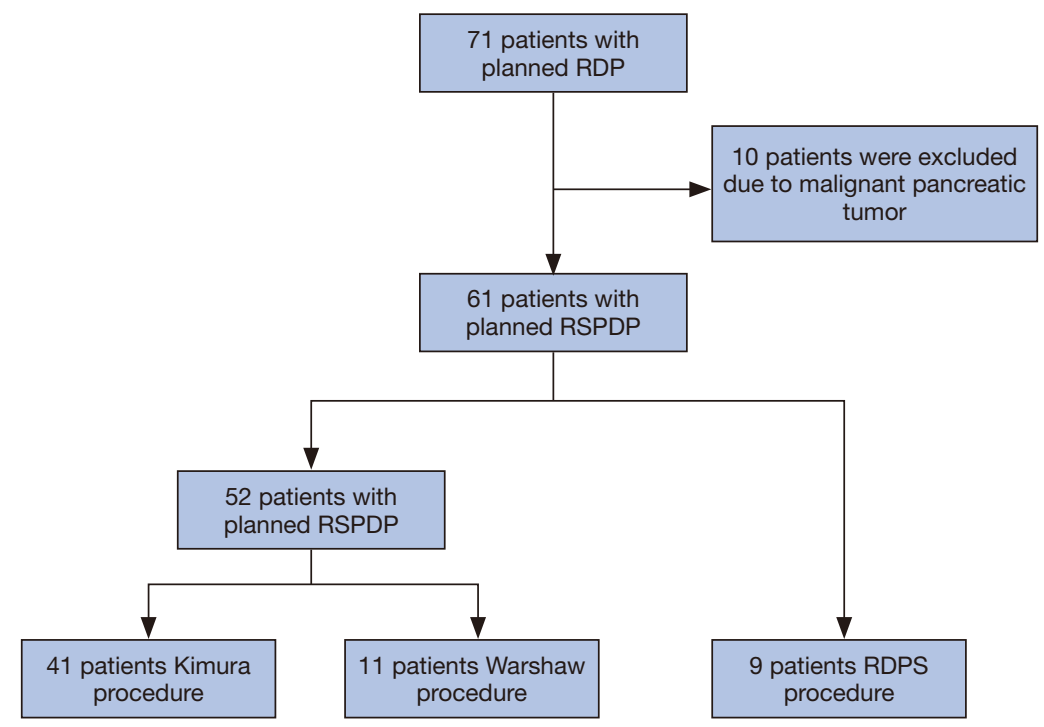

Figure 5 Flow chart of the patients with planned RDP.

vs. $12.2 \%, \mathrm{P}=0.015)$. There were no significant differences concerning other clinicopathologic characteristics or perioperative and postoperative factors between the two groups $(\mathrm{P}>0.05)$.

\section{Comparison between RDP with or without splenic vessel preservation}

To evaluate the efficacy of the robotic system for splenic vessel preservation during RDP, the clinicopathologic characteristics and perioperative and postoperative factors of the patients who underwent RDP with or without splenic vessel preservation were analyzed (the Kimura procedure $v s$. the Warshaw procedure and RDPS) (Table 3). Significant differences in sex $(\mathrm{P}=0.022)$ and radiographic appearance $(\mathrm{P}=0.028)$ were noted between the vessel preservation and non-preservation groups. There were larger proportions of female patients (70.7\% vs. $40.0 \%)$ and cystic tumors $(65.9 \%$ vs. 30.0\%) in the former group than in the latter group. The non-preservation group had a remarkably higher incidence of tumors $\geq 6 \mathrm{~cm}(45.0 \%$ vs. $17.1 \%, \mathrm{P}=0.030)$, obvious splenic vessel compression by the tumor $(65.0 \%$ vs. $12.2 \%$, $\mathrm{P}=0.000$ ), and more EBL (median 200 vs. $50 \mathrm{~mL}, \mathrm{P}=0.000$ ) than the vessel preservation group. Other clinicopathologic characteristics and perioperative and postoperative factors showed no significant differences between the two groups $(\mathrm{P}>0.05)$.

\section{Risk factors for RDP with splenic preservation}

Three logistic regression models with univariate and multivariate analyses were created to identify risk factors for RDP with splenic preservation (RSPDP vs. RDPS, $\mathrm{n}=61$ ), RSPDP with splenic vessel preservation (Kimura $v$ s. Warshaw, n=52), and RDP with splenic vessel preservation (vessel preservation $v s$. non-preservation, $\mathrm{n}=61$ ). In the first logistic regression model for RDP with splenic preservation, sex, tumor size and splenic vessel compression of the tumor were included in the multivariate analysis. Although tumor size and splenic vessel compression of the tumor showed significant differences in the univariate analysis, they showed no significant differences in the multivariate analysis (Table 4).

\section{Risk factors for RSPDP with splenic vessel preservation}

In the second logistic regression model for RSPDP with splenic vessel preservation, age, sex, tumor size, radiographic appearance and splenic vessel compression by the tumor were included in the multivariate analysis. The results of the multivariate analysis showed that age $\geq 50$ years (vs. age $<50$ years; OR $0.078,95 \%$ CI: $0.007-0.888, \mathrm{P}=0.040$ ) and obvious splenic vessel compression by the tumor (vs. no/ slight splenic vessel compression by the tumor; OR 0.021, 95\% CI: $0.002-0.271, \mathrm{P}=0.003$ ) were significant predictors 
Table 1 Comparison between RSPDP and RDPS

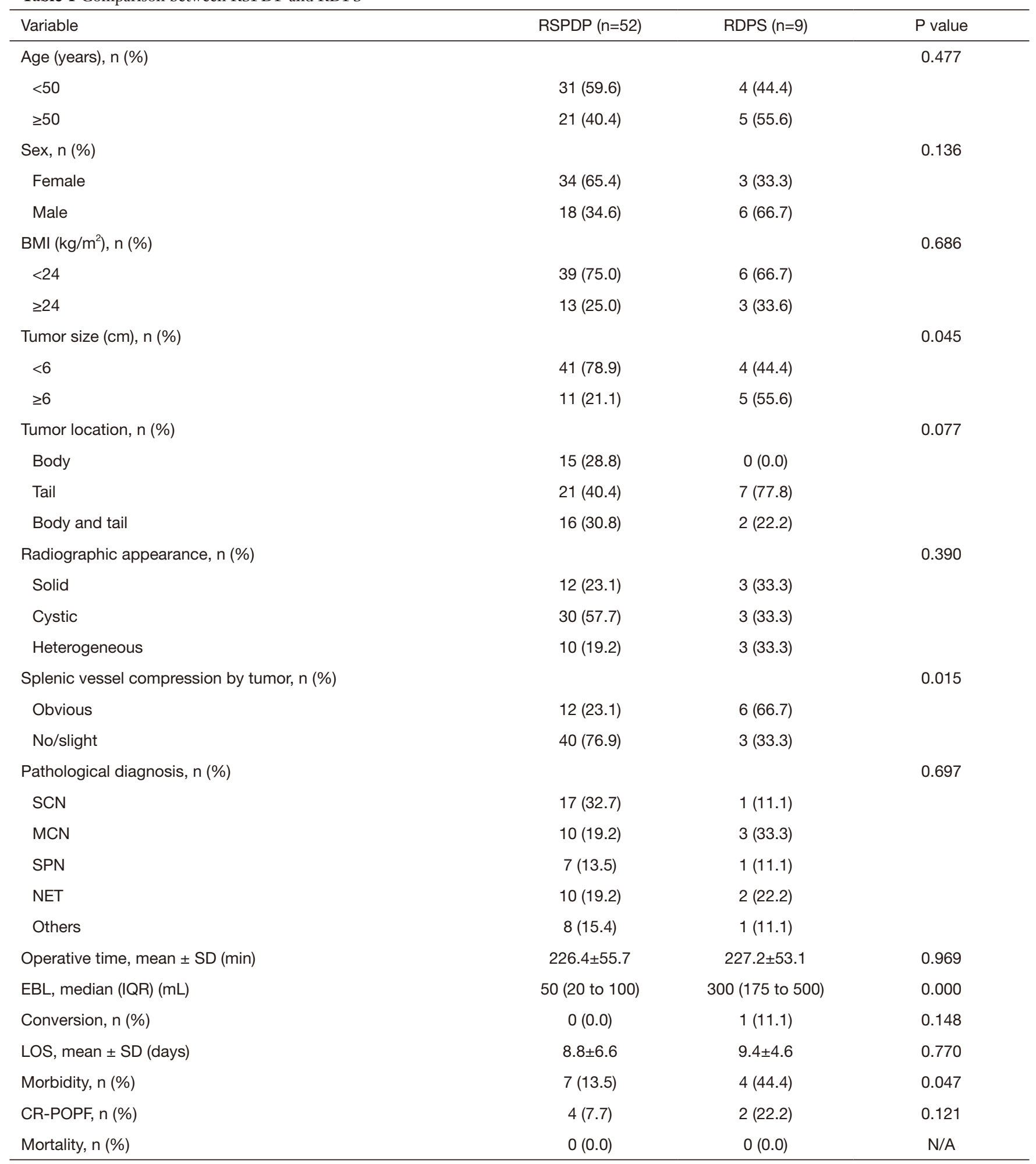

RDP, robotic splenic preservation distal pancreatectomy; RSPDP, robotic splenic preservation distal pancreatectomy; RDPS, robotic distal pancreatosplenectomy; BMI, body mass index; SCN, serous cystic neoplasm; MCN, mucinous cystic neoplasm; SPN, solid pseudopapillary neoplasm; NET, neuroendocrine tumor; EBL, estimated blood loss; LOS, length of stay; CR-POPF, clinically relevant postoperative pancreatic fistula; N/A, not applicable. 
Table 2 Comparison between the Kimura and Warshaw technique

\begin{tabular}{|c|c|c|c|}
\hline Variable & Kimura $(n=41)$ & Warshaw $(n=11)$ & $P$ value \\
\hline$<50$ & $27(65.9)$ & $4(36.4)$ & \\
\hline$\geq 50$ & $14(34.1)$ & $7(63.6)$ & \\
\hline Sex, n (\%) & & & 0.159 \\
\hline BMI $\left(\mathrm{kg} / \mathrm{m}^{2}\right), \mathrm{n}(\%)$ & & & 0.435 \\
\hline$<24$ & $32(78.0)$ & $7(63.6)$ & \\
\hline$\geq 24$ & $9(22.0)$ & $4(36.4)$ & \\
\hline Tumor size (cm), n (\%) & & & 0.216 \\
\hline Tumor location, n (\%) & & & 0.453 \\
\hline Body & $12(29.3)$ & $3(27.3)$ & \\
\hline Tail & $18(43.9)$ & $3(27.3)$ & \\
\hline Body and tail & $11(26.8)$ & $5(45.4)$ & \\
\hline Radiographic appearance, n (\%) & & & 0.000 \\
\hline Solid & $8(19.5)$ & $4(36.4)$ & \\
\hline Cystic & $27(65.9)$ & $3(27.3)$ & \\
\hline Heterogeneous & $6(14.6)$ & $4(36.4)$ & \\
\hline MCN & $8(19.5)$ & $2(18.2)$ & \\
\hline SPN & $5(12.2)$ & $2(18.2)$ & \\
\hline NET & $6(14.6)$ & $4(36.4)$ & \\
\hline Others & $8(19.5)$ & $0(0.0)$ & \\
\hline Operative time, mean \pm SD (min) & $226.0 \pm 56.1$ & $228.2 \pm 56.7$ & 0.909 \\
\hline EBL, median (IQR) (mL) & 50 (20 to 100$)$ & 50 (20 to 100$)$ & 0.563 \\
\hline Conversion, n (\%) & $0(0.0)$ & $0(0.0)$ & N/A \\
\hline LOS, mean \pm SD (days) & $8.2 \pm 6.8$ & $10.8 \pm 5.6$ & 0.250 \\
\hline Morbidity, n (\%) & $5(12.2)$ & $2(18.2)$ & 0.630 \\
\hline CR-POPF, n (\%) & $3(7.3)$ & $1(9.1)$ & 1.000 \\
\hline Mortality, n (\%) & $0(0.0)$ & $0(0.0)$ & $\mathrm{N} / \mathrm{A}$ \\
\hline
\end{tabular}

RSPDP, robotic splenic preservation distal pancreatectomy; BMI, body mass index; SCN, serous cystic neoplasm; MCN, mucinous cystic neoplasm; SPN, solid pseudopapillary neoplasm; NET, neuroendocrine tumor; EBL, estimated blood loss; LOS, length of stay; CR-POPF, clinically relevant postoperative pancreatic fistula; N/A, not applicable. 
Table 3 Comparison between RDP with or without splenic vessel preservation (Kimura vs. Warshaw + RDPS)

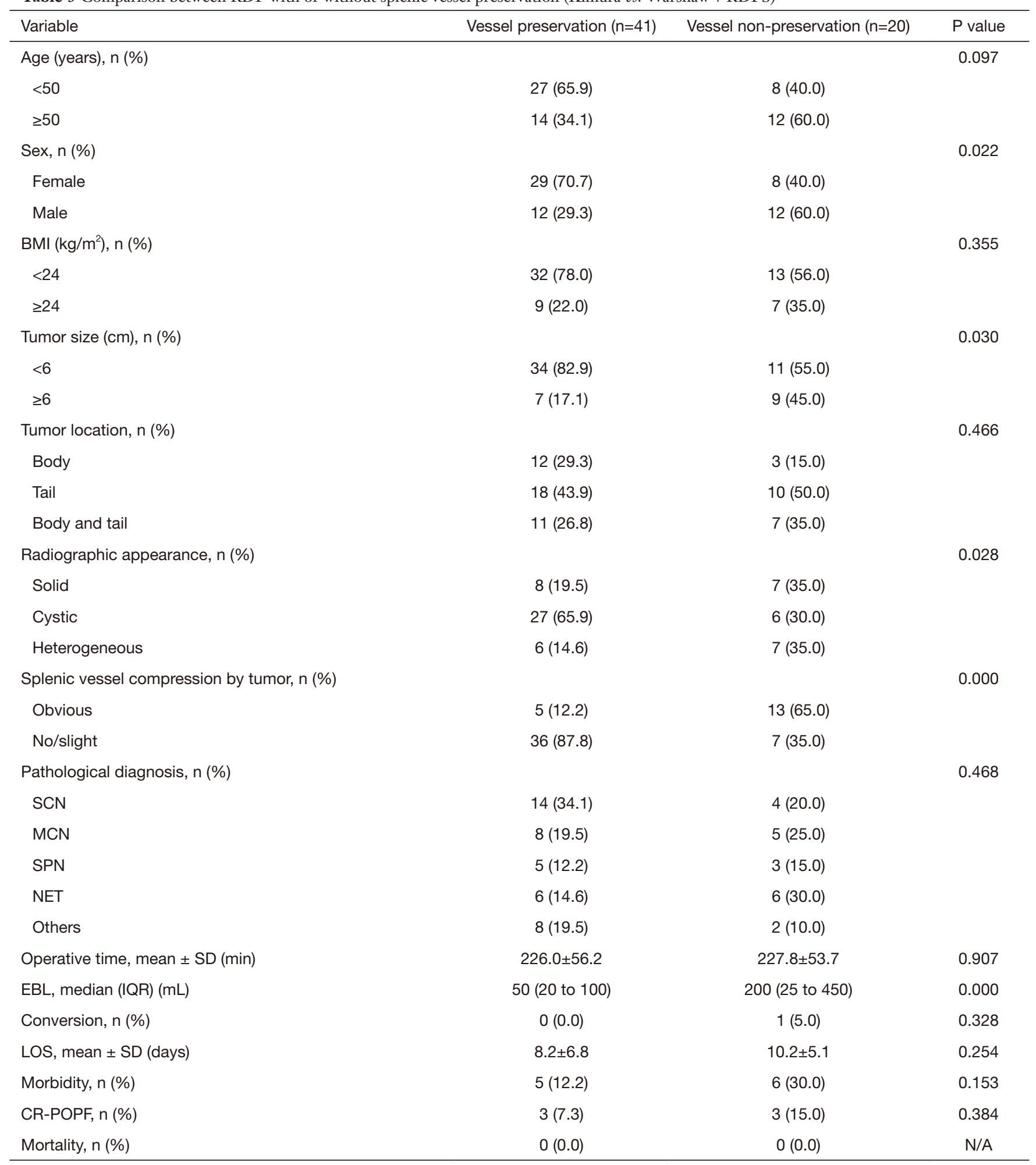

RDP, robotic splenic preservation distal pancreatectomy; RDPS, robotic distal pancreatosplenectomy; BMI, body mass index; SCN, serous cystic neoplasm; MCN, mucinous cystic neoplasm; SPN, solid pseudopapillary neoplasm; NET, neuroendocrine tumor; EBL, estimated blood loss; LOS, length of stay; CR-POPF, clinically relevant postoperative pancreatic fistula; N/A, not applicable. 
Table 4 Risk factors for RDP with splenic preservation (RSPDP $v$ s. RDPS, $\mathrm{n}=61$ )

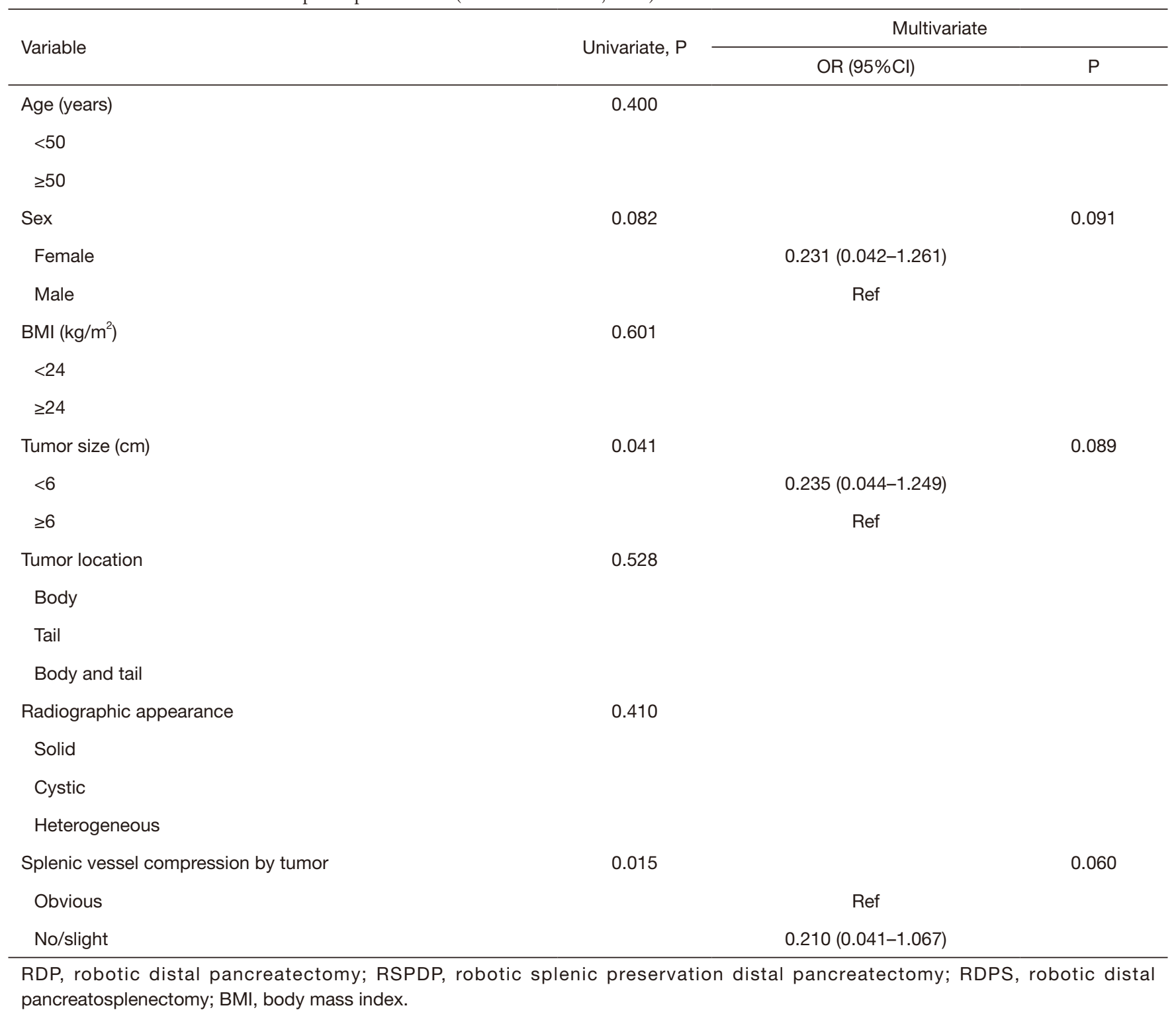

of splenic vessel preservation with RSPDP and (Table 5).

\section{Risk factors for RDP with splenic vessel preservation}

In the third logistic regression model for RDP with splenic vessel preservation, age, sex, tumor size, radiographic appearance and splenic vessel compression by the tumor were included in the multivariate analysis. The results of the multivariate analysis showed that age $\geq 50$ years ( $v s$. age $<50$ years; OR $0.136,95 \%$ CI: $0.021-0.883, \mathrm{P}=0.037)$ and obvious splenic vessel compression by the tumor (vs. no/ slight splenic vessel compression by the tumor; OR 0.019 , 95\% CI: 0.002-0.176, $\mathrm{P}=0.000$ ) were significant predictors of splenic vessel preservation with RDP (Table 6).

\section{Discussion}

In recent decades, spleen-preserving MIDP has been advocated for benign or low-grade malignant pancreatic tumors (17). One important reason is that the spleen plays a vital role in the immune defense (18). Splenectomy is associated with increased risks of infection and a 
Table 5 Risk factors for RSPDP with splenic vessel preservation (Kimura vs. Warshaw, n=52)

\begin{tabular}{|c|c|c|c|}
\hline Variable & Univariate, $\mathrm{P}$ & \multicolumn{2}{|c|}{ Multivariate } \\
\hline Age (years) & & & 0.040 \\
\hline$<50$ & 0.086 & $0.078(0.007-0.888)$ & \\
\hline$\geq 50$ & & Ref & \\
\hline Female & & $0.751(0.081-6.939)$ & \\
\hline Male & & Ref & \\
\hline BMI $\left(\mathrm{kg} / \mathrm{m}^{2}\right)$ & 0.333 & & \\
\hline \multicolumn{4}{|l|}{$<24$} \\
\hline$<6$ & & $0.383(0.044-3.325)$ & \\
\hline$\geq 6$ & & Ref & \\
\hline Tumor location & 0.465 & & \\
\hline \multicolumn{4}{|l|}{ Body } \\
\hline \multicolumn{4}{|l|}{ Tail } \\
\hline \multicolumn{4}{|l|}{ Body and tail } \\
\hline Radiographic appearance & 0.089 & & 0.168 \\
\hline Solid & & Ref & \\
\hline
\end{tabular}

RSPDP, robotic splenic preservation distal pancreatectomy; BMI, body mass index.

significantly elevated postoperative platelet count with hypercoagulability. Addeo et al. reported that minimally invasive DPS was associated with a higher incidence of SV thrombosis than SPDP $(81.8 \%$ vs. $25 \%, \mathrm{P}<0.001)(19)$. Among the spleen-preserving techniques, the Warshaw technique, which involves segment resection of the splenic vessels, is easy to perform (20) but has increased risks of splenic infarction and perigastric varices as well as secondary bleeding (21). Louis et al. reported that 10 of 48 patients (27\%) who underwent SPDP with the Warshaw technique developed perigastric varices during a median follow-up period of 76 months (22). Compared with the Warshaw technique, the Kimura technique has fewer complications because of complete preservation of the splenic vessels but is time consuming and technically challenging.

In the era of advanced minimally invasive pancreatic surgery, some existing studies have shown equivalent outcomes of laparoscopic and robotic surgeries $(10,23)$. However, compared to LDP, RDP shows advantages in both splenic and splenic vessel preservation $(24,25)$. These advantages may be attributed to the high flexibility of robotic instruments and precise suturing both in the dissection of splenic vessels and in the control of bleeding of the splenic vessels. Chen et al. 
Table 6 Risk factors for RDP with splenic vessel preservation (vessel preservation $v$ s. non-preservation, $\mathrm{n}=61$ )

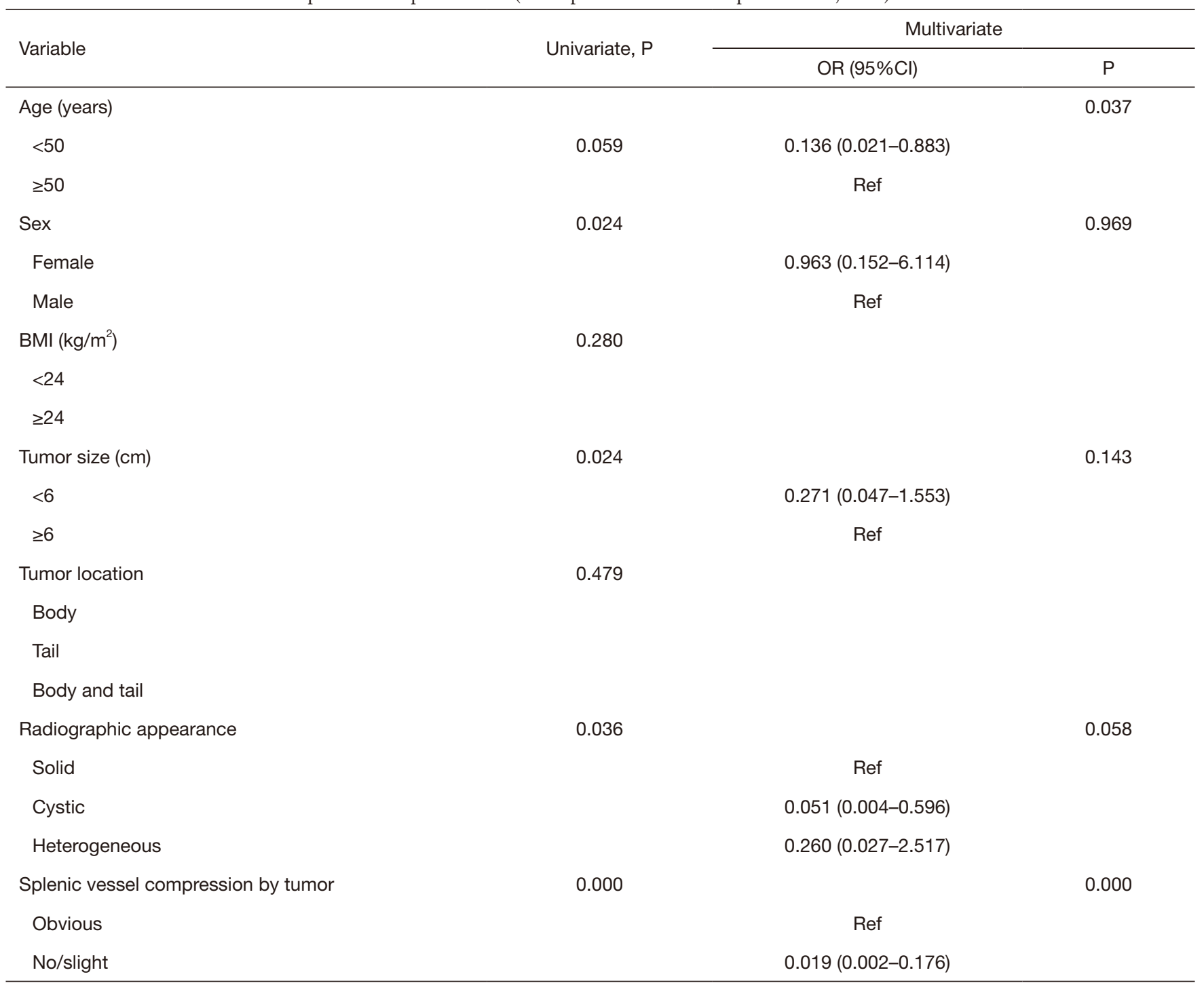

RDP, robotic distal pancreatectomy; BMI, body mass index.

reported a remarkably higher incidence of overall splenic preservation $(95.7 \%$ vs. $39.4 \%, \mathrm{P}<0.001)$ and Kimura splenic preservation $(72.3 \%$ vs. $21.2 \%, \mathrm{P}<0.001)$ with RDP than with LDP (26). Hong et al. reported that RDP was associated with a higher rate of splenic preservation than LDP (96.8\% vs. $82.5 \%, \mathrm{P}=0.02)(14)$. The splenic preservation rate in the present study was $85.2 \%$. Additionally, the rate of splenic vessel preservation with the Kimura technique was $78.8 \%$. The rates of splenic preservation and splenic vessel preservation were similar to those in previous studies in high-volume pancreatic centers. In the three-step strategy of splenic preservation for planned RSPDP, the Kimura technique was first attempted whenever spleen vessel preservation was possible. The Warshaw technique was considered an alternative method of spleen preservation under the condition of long-segment compression of the splenic vessels by the tumor or uncontrolled expected bleeding of the splenic vessels. RDPS was performed if the two spleen-preserving techniques failed. Choi et al. reported that initially planned laparoscopic distal pancreatosplenectomy had a shorter operative time than laparoscopic spleen-preserving distal pancreatectomy (18). However, no significant differences in the operative time between these three procedures were 
noted in our study, which might be attributed to the stepby-step strategy. However, the RSPDP group still had less EBL than the RDPS group. The high splenic preservation rate and splenic vessel preservation rate in the present study demonstrate the feasibility and efficacy of the three-step strategy for splenic preservation with RSPDP. Based on the results of the multivariate analysis, obvious splenic vessel compression by the tumor might suggest that the patient receive the Warshaw procedure or RDPS.

Predictive risk factors for splenic preservation or splenic vessel preservation are of great importance in surgical decision-making in MIDP. However, only a few studies have focused on this issue. Dai et al. reported that a tumor size at a cut-off value of $3 \mathrm{~cm}$ was an independent risk factor for identifying splenic vessel preservation with planned laparoscopic splenic vessel preservation operations (area under the curve $0.724,95 \%$ CI: $0.63-0.82, \mathrm{P}<0.01$ ), especially for tumors located in the pancreatic body (27). A propensity score-matched study by the Minimally Invasive Liver and Pancreatic Surgery Study Group-UK that enrolled 456 patients reported that a tumor size $\geq 3 \mathrm{~cm}$ was a significant factor for unplanned splenectomy with LDP (OR 2.28, 95\% CI: 1.16-4.48, $\mathrm{P}=0.02)$ (28). In the present study, a tumor size at a cut-off value of $6 \mathrm{~cm}$ showed significant differences in the comparison of RDP with or without splenic preservation, as well as RDP with or without splenic vessel preservation. Tumor size was not significantly different according to the multivariate logistic regression models. However, obvious splenic vessel compression by the tumor showed significant differences in all the comparisons of splenic and splenic vessel preservation (Tables 1,2,3). It also showed significant differences in the second and third logistics models in contrast to splenic vessel preservation with planned RDP and RSPDP. Therefore, obvious splenic vessel compression by the tumor is an independent risk factor for splenic vessel preservation with planned RDP. The mean age of all patients was $45.8 \pm 14.9$ years. Therefore, all patients were divided into two subgroups according to age: $<50$ and $\geq 50$ years. Interestingly, the logistic regression models showed that age $\geq 50$ years was also an independent risk factor for splenic vessel preservation with RSPDP (OR 0.078, 95\% CI: 0.007-0.888, $\mathrm{P}=0.040$ ) and RDP (OR 0.136, 95\% CI: 0.021-0.883, $\mathrm{P}=0.037$ ).

The perioperative outcomes of RDP were acceptable in the present study, indicating the efficacy of the procedure. The overall rate of CR-POPF (grade B) in the study was $9.8 \%$. No significant difference concerning the incidence of CR-POPF between the three procedures was noted (Kimura, 7.3\%; Warshaw, 9.1\%; and RDPS, 22.2\%;
$\mathrm{P}>0.05)$. A recent international, multi-institutional study that enrolled 2,026 patients in 10 centers by Ecker et al. showed that the CR-POPF rate after pancreatic transection with the stapler technique was $12.7 \%$, which was in accordance with that in the present study (29). It is crucial to select a suitable stapler for pancreatic transection according to the thickness of the pancreas. The Kimura technique, which involves complete preservation of the splenic vessels, theoretically increases the risks of postoperative bleeding, especially in the case of concomitant POPF. However, no postoperative intra-abdominal hemorrhage was observed in the current study. This observation may be attributed to the precise suturing of most of the branches of the splenic vessels coursing into the pancreas with 5-0 Prolene sutures, which are more effective than vascular clips for preventing postoperative bleeding, with RSPDP. The use of routine or selective intra-abdominal drainage after DP remains debatable. A previous RCT by Van Buren et al. (30) showed no significant differences in the incidence of CR-POPF between patients with and without routine intraperitoneal drainage after DP (18\% vs. $12 \%, \mathrm{P}>0.05)$. The latter group of patients was associated with a higher rate of intra-abdominal fluid collection ( $9 \%$ vs. $22 \%, \mathrm{P}=0.0004)$. However, no intra-abdominal fluid collection was noted in the present study, indicating the need for routine drainage.

This study had some limitations. First, it was a retrospective cohort analysis with a single surgeon's experience in a single institution. Second, there were only a few patients in the RSPDP group with the Warshaw technique and in the RDPS group, which might have resulted in statistical bias. However, this finding might partly be attributed to the high splenic preservation rate in the RSPDP group with the Kimura technique. Third, the RDP cohort included a learning curve. A larger sample size is required in the future to verify the efficacy of the robotic system in SPDP.

\section{Conclusions}

The "Kimura-first" strategy is feasible and safe for RSPDP, with high rates of splenic and splenic vessel preservation. Obvious splenic vessel compression by the tumor can be used as a predictor of splenic vessel preservation with planned RSPDP.

\section{Acknowledgments}

Funding: This study was supported by the Medical 
Minimally Invasive Center Program of Fujian Province, China (No. 2017-171), the Joint Funds of Scientific and Technological Innovation Program of Fujian Province (No. 2017Y9059), and the Key Clinical Specialty Discipline Construction Program of Fujian Province, China (No. 2012-649).

\section{Footnote}

Reporting Checklist: The authors have completed the STROBE reporting checklist. Available at http://dx.doi. org/10.21037/gs-20-576

Data Sharing Statement: Available at http://dx.doi. org/10.21037/gs-20-576

Peer Review File: Available at http://dx.doi.org/10.21037/gs20-576

Conflicts of Interest: All authors have completed the ICMJE uniform disclosure form (available at http://dx.doi. org/10.21037/gs-20-576). The authors have no conflicts of interest to declare.

Ethical Statement: The authors are accountable for all aspects of the work in ensuring that questions related to the accuracy or integrity of any part of the work are appropriately investigated and resolved. The study was conducted in accordance with the Declaration of Helsinki (as revised in 2013). Written informed consent was obtained from the patients for publication of this manuscript and any accompanying images. The study protocol was approved by the ethics committee of Fujian Medical University Union Hospital (2020KY0107).

Open Access Statement: This is an Open Access article distributed in accordance with the Creative Commons Attribution-NonCommercial-NoDerivs 4.0 International License (CC BY-NC-ND 4.0), which permits the noncommercial replication and distribution of the article with the strict proviso that no changes or edits are made and the original work is properly cited (including links to both the formal publication through the relevant DOI and the license). See: https://creativecommons.org/licenses/by-nc-nd/4.0/.

\section{References}

1. Ielpo B, Duran H, Diaz E, et al. Robotic versus laparoscopic distal pancreatectomy: A comparative study of clinical outcomes and costs analysis. Int J Surg 2017;48:300-4.

2. Tran Cao HS, Lopez N, Chang DC, et al. Improved perioperative outcomes with minimally invasive distal pancreatectomy: results from a population-based analysis. JAMA Surg 2014;149:237-43.

3. Alfieri S, Butturini G, Boggi U, et al. Short-term and longterm outcomes after robot-assisted versus laparoscopic distal pancreatectomy for pancreatic neuroendocrine tumors (pNETs): a multicenter comparative study. Langenbecks Arch Surg 2019;404:459-68.

4. de Rooij T, van Hilst J, van Santvoort H, et al. Minimally Invasive Versus Open Distal Pancreatectomy (LEOPARD): A Multicenter Patient-blinded Randomized Controlled Trial. Ann Surg 2019;269:2-9.

5. Lee LS, Hwang HK, Kang CM, et al. Minimally Invasive Approach for Spleen-Preserving Distal Pancreatectomy: a Comparative Analysis of Postoperative Complication Between Splenic Vessel Conserving and Warshaw's Technique. J Gastrointest Surg 2016;20:1464-70.

6. Kim S, Yoon YS, Han HS, et al. A blunt dissection technique using the LigaSure vessel-sealing device improves perioperative outcomes and postoperative splenic-vessel patency after laparoscopic spleen- and splenic-vessel-preserving distal pancreatectomy. Surg Endosc 2018;32:2550-8.

7. Kang CM, Kim DH, Lee WJ, et al. Conventional laparoscopic and robot-assisted spleen-preserving pancreatectomy: does da Vinci have clinical advantages? Surg Endosc 2011;25:2004-9.

8. Kimura W, Yano M, Sugawara S, et al. Spleen-preserving distal pancreatectomy with conservation of the splenic artery and vein: techniques and its significance. J Hepatobiliary Pancreat Sci 2010;17:813-23.

9. Warshaw AL. Conservation of the spleen with distal pancreatectomy. Arch Surg 1988;123:550-3.

10. Kamarajah SK, Sutandi N, Robinson SR, et al. Robotic versus conventional laparoscopic distal pancreatic resection: a systematic review and meta-analysis. HPB (Oxford) 2019;21:1107-18.

11. Eckhardt S, Schicker C, Maurer E, et al. RoboticAssisted Approach Improves Vessel Preservation in Spleen-Preserving Distal Pancreatectomy. Dig Surg 2016;33:406-13.

12. Yang SJ, Hwang HK, Kang CM, et al. Revisiting the potential advantage of robotic surgical system in spleenpreserving distal pancreatectomy over conventional 
laparoscopic approach. Ann Transl Med 2020;8:188.

13. Alfieri S, Boggi U, Butturini G, et al. Full Robotic Distal Pancreatectomy: Safety and Feasibility Analysis of a Multicenter Cohort of 236 Patients. Surg Innov 2020;27:11-8.

14. Hong S, Song KB, Madkhali AA, et al. Robotic versus laparoscopic distal pancreatectomy for left-sided pancreatic tumors: a single surgeon's experience of 228 consecutive cases. Surg Endosc 2020;34:2465-73.

15. Liu R, Liu Q, Zhao ZM, et al. Robotic versus laparoscopic distal pancreatectomy: A propensity score-matched study. J Surg Oncol 2017;116:461-9.

16. Bassi C, Marchegiani G, Dervenis C, et al. The 2016 update of the International Study Group (ISGPS) definition and grading of postoperative pancreatic fistula: 11 Years After. Surgery 2017;161:584-91.

17. Nakata K, Shikata S, Ohtsuka T, et al. Minimally invasive preservation versus splenectomy during distal pancreatectomy: a systematic review and meta-analysis. J Hepatobiliary Pancreat Sci 2018;25:476-88.

18. Choi SH, Seo MA, Hwang HK, et al. Is it worthwhile to preserve adult spleen in laparoscopic distal pancreatectomy? Perioperative and patient-reported outcome analysis. Surg Endosc 2012;26:3149-56.

19. Addeo P. Splenic vein thrombosis and pancreatic fistula after minimally invasive distal pancreatectomy (Br J Surg 2014; 101: 114-119). Br J Surg 2014;101:119-20.

20. Wang L, Wu D, Cheng YG, et al. Warshaw Technique in Laparoscopic Spleen-Preserving Distal Pancreatectomy: Surgical Strategy and Late Outcomes of Splenic Preservation. Biomed Res Int 2019;2019:4074369.

21. Kim HS, Park JS, Yoon DS. True learning curve of laparoscopic spleen-preserving distal pancreatectomy with splenic vessel preservation. Surg Endosc 2019;33:88-93.

22. Louis D, Alassiri A, Kirzin S, et al. Gastric bleeding risk

Cite this article as: Lin $\mathrm{X}$, Lin $\mathrm{R}$, Lu F, Yang Y, Wang C, Fang H, Huang H. "Kimura-first" strategy for robotic spleen-preserving distal pancreatectomy: experiences from 61 consecutive cases in a single institution. Gland Surg 2021;10(1):186-200. doi: 10.21037/gs-20-576 following spleen preserving distal pancreatectomy with excision of the splenic vessels: a long-term follow-up. HPB (Oxford) 2017;19:345-51.

23. Kamarajah SK, Bundred J, Marc OS, et al. Robotic versus conventional laparoscopic pancreaticoduodenectomy a systematic review and meta-analysis. Eur J Surg Oncol 2020;46:6-14.

24. Marino MV, Mirabella A, Gomez Ruiz M, et al. RoboticAssisted versus Laparoscopic Distal Pancreatectomy: The Results of a Case-Matched Analysis from a Tertiary Care Center. Dig Surg 2020;37:229-39.

25. Lyman WB, Passeri M, Sastry A, et al. Roboticassisted versus laparoscopic left pancreatectomy at a high-volume, minimally invasive center. Surg Endosc 2019;33:2991-3000.

26. Chen S, Zhan Q, Chen JZ, et al. Robotic approach improves spleen-preserving rate and shortens postoperative hospital stay of laparoscopic distal pancreatectomy: a matched cohort study. Surg Endosc 2015;29:3507-18.

27. Dai MH, Shi N, Xing C, et al. Splenic preservation in laparoscopic distal pancreatectomy. Br J Surg 2017;104:452-62.

28. Moekotte AL, Lof S, White SA, et al. Splenic preservation versus splenectomy in laparoscopic distal pancreatectomy: a propensity score-matched study. Surg Endosc 2020;34:1301-9.

29. Ecker BL, McMillan MT, Allegrini V, et al. Risk Factors and Mitigation Strategies for Pancreatic Fistula After Distal Pancreatectomy: Analysis of 2026 Resections From the International, Multi-institutional Distal Pancreatectomy Study Group. Ann Surg 2019;269:143-9.

30. Van Buren G, 2nd, Bloomston M, Schmidt CR, et al. A Prospective Randomized Multicenter Trial of Distal Pancreatectomy With and Without Routine Intraperitoneal Drainage. Ann Surg 2017;266:421-31. 